\title{
EXPERIMENTAL VALIDATION OF METAMODELS FOR INTELLIGENT AGENTS IN CONFLICT
}

\author{
James MOFFAT and Susan WITTY
}

\section{Introduction}

As discussed in a previous paper, ${ }^{1}$ we are attempting to develop mathematical 'metamodels' of agent-based simulation models. These meta-models fall within the area of what is loosely referred to as complexity theory, and exploit the mathematical approaches which are being developed to gain understanding of natural non-linear systems (such as ecosystems of species, or the physics of spin systems). Such an approach is most likely to be relevant to future command and control structures such as Network Centric Warfare. ${ }^{2}$ In particular, Moffat discusses in detail the connection between the mathematics of complexity and the likely emergent behaviour of future command and control driven 'Information Age' conflict. ${ }^{3}$

\section{Cellular Automata}

As a means of gaining understanding, we have carried out a number of experiments using simple cellular automata based models that are relevant to conflict. Such models have been developed in response to the theory that human conflict is a complex, non-linear system, ${ }^{4}$ which in dynamical system terms, occurs far from equilibrium. In other areas of research on the complexity of natural systems, such cellular automata models have been used to identify key emergent properties of such systems. 5

The 'new sciences' of complexity and chaos, although not yet fully developed into a coherent theory, provide a way of looking at such interacting agents in conflict. ${ }^{6}$ In this paper we first show in detail that historical data indicate the existence of a fractal attractor for at least some types of conflict (based on initial work by Lauren ${ }^{7}$ ). We then show that experimental data from runs of such simple cellular automata models supports the hypotheses, which can be derived from our theoretical meta-models of the process. 
The experimental data presented here have been derived from a particular cellular automata model called ISAAC ${ }^{8}$ which was developed for the US Marine Corps as part of their 'Project Albert' research initiative.'

\section{An Attractor for Conflict}

For non-linear dynamical systems, we know that there are only a small set of possible attractors for the dynamics of the system typically corresponding to a stable invariant final state, a limit cycle (corresponding to a periodic final state), and a 'strange attractor' (normally a fractal set), corresponding to a chaotic state. ${ }^{10}$

Lauren indicates that for at least some conflict situations, the dynamics of interaction of the forces evolves towards an attractor state, which is independent of the initial conditions. ${ }^{11}$ The idea is that an essentially straight line frontage between two tactical opponents will buckle into a fractal shape, whose fractal dimension can be calculated as a function of the force ratio of the forces involved, (the number of attackers to the number of defenders), as derived from Historical Analysis of infantry battles carried out by the UK Defence Science and Technology Laboratory (DSTL). Lauren uses as a basis for his approach a regression analysis of historical tactical level conflicts carried out by DSTL, which indicates that the non-dimensional parameter:

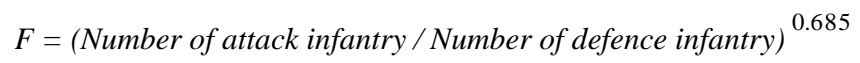

is a multiplier for the base number of casualties of the attacking force per defence weapon. Note that from a previous work of Moffat we expect powers of nondimensional parameters to be of key importance in such 'meta-models' of the process. ${ }^{12}$ In fact, from the same work of the author we can say that this is a metamodel of Type 2, since the exponent cannot be derived solely from dimensionality considerations. As a consequence of this, Lauren was able to shows that the combat front will buckle over time, and in the limit will have a fractal dimension $D=1.685$. Thus for this type of conflict, the dynamics in the time invariant state are similar to those of a chaotic system.

In this case we can derive the underlying dynamics producing this statistical effect. It turns out that this fractal factor is due fundamentally to detection of targets, ${ }^{13}$ and comes from a model of the engagement process, which leads to the following relationship:

$$
\frac{1}{R}=\frac{k_{1}}{T}+k_{2},
$$


where $k_{1}$ and $k_{2}$ are constants, $R$ is the defender rate of fire and $T$ is the number of targets in view. ${ }^{14}$

It reflects the asymmetry of the infantry battle in the following sense. ${ }^{15}$ The attack force aim is to close on the defence position, and fire is used in a general suppressive mode - actual casualties caused to the defence are only a small part of the process at this point. However, from the defence perspective, the aim is to deter the attack, and casualties to the attack force are very important. Such casualties to the attack force are a direct reflection of the inter-visibility of targets to the defence force as discussed above.

As with most applications of fractal processes, the process breaks down at some point due to the granularity of the resolution. In this case, the process remains valid up to about 30 meters closing distance between the attack and defence. At that point a different mechanism comes into play, leading to local defence surrender and attack overrun of defence positions. ${ }^{16}$

More generally, the figure of 0.685 relates to open terrain. In urban areas it is about $0.5,{ }^{17}$ giving rise to a fractal dimension of 1.5 for the attractor state. The closing to overrun appears to occur differently in urban and wooded terrain as compared with open terrain. ${ }^{18}$ For example, in open conditions, the closing part of the battle occurs across the front. By contrast, in urban conditions, the attack force is split into small subunits that individually close on defence locations leading to local surrender and overrun.

In terms of modelling such a process using cellular automata, if we assume that this process is akin to the cellular automata model of 'invasion percolation' in which one fluid is invaded by another in a porous medium, the fractal dimension of the boundary of the resulting interface lies in the range $1.33-1.89,{ }^{19}$ which agrees with our experimental data range (based on historical conflict regression analysis) of 1.5-1.685 for the fractal dimension of our attractor set.

\section{Local Clustering}

In order to analyse the 'swarming' dynamics of cluster formation and dissolution in the ISAAC cellular automata model of conflict, first we need to consider how to define such a cluster. In Theoretical Physics, it is usual to define neighbouring agents as those, which are North, South, East or West, adjacent to the agent in question, known as 'nearest neighbour' clustering. For a single time step in any run of a cellular automata based model, the number and size of clusters of agents can be determined using the Hoshen-Kopelman algorithm. ${ }^{20}$ 


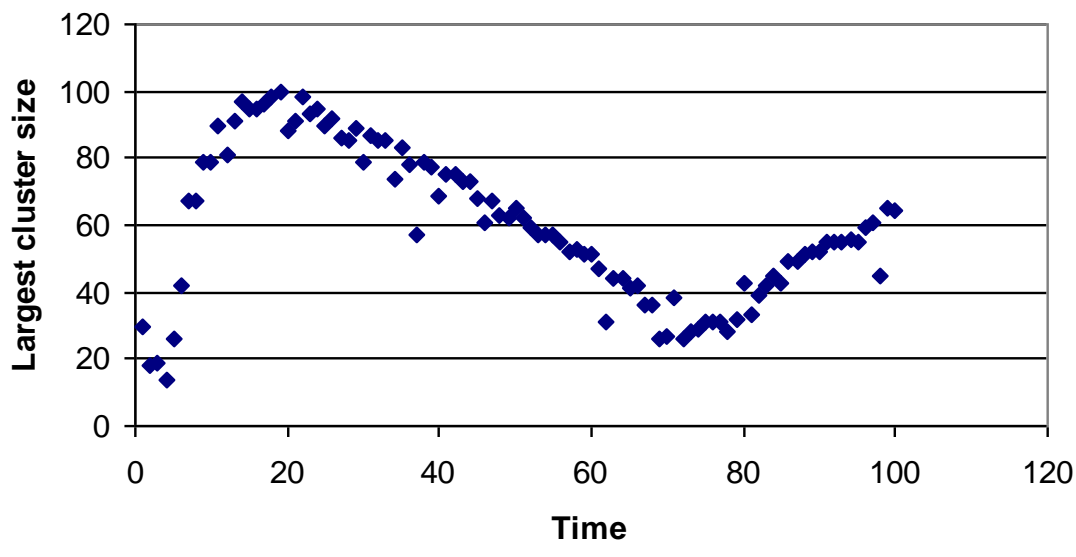

Figure 1: Evolution of the Largest Red Cluster Size within the Scenario (Red Successful).

This algorithm has been applied to a particular 'scenario' in the ISAAC model in which the Red agents attempt, by a swarming strategy, to reach a high value objective defended by a smaller number of Blue agents. The particular interest of this scenario is that in all stochastic replications (over 100 in total) of the model except one, the Red agents reach and take control of the high value objective. In the exceptional case, Blue is able to defend this objective successfully.

Once the cluster numbers and sizes can be determined for each experimental replication of the scenario, there are a number of ways to analyse the data. First we look at is the size of the largest cluster. This gives an indication of the ability of the agents to cluster or the amount of dispersal of the agents. For example, if the largest cluster size is near to the total number of agents, we know that that is the only cluster. However, if the largest cluster is small, then we know that the agents are dispersed in many small clusters.

Figure 1 shows a typical evolution of the largest cluster size for the Red agents, in the case where Red is successful in taking control of the high value objective. Each break in the slope of this plot corresponds to a new phase in the operation - first the move to infiltration and engagement with Blue agents, secondly the infiltration and engagement phase, and finally clustering around the objective. 


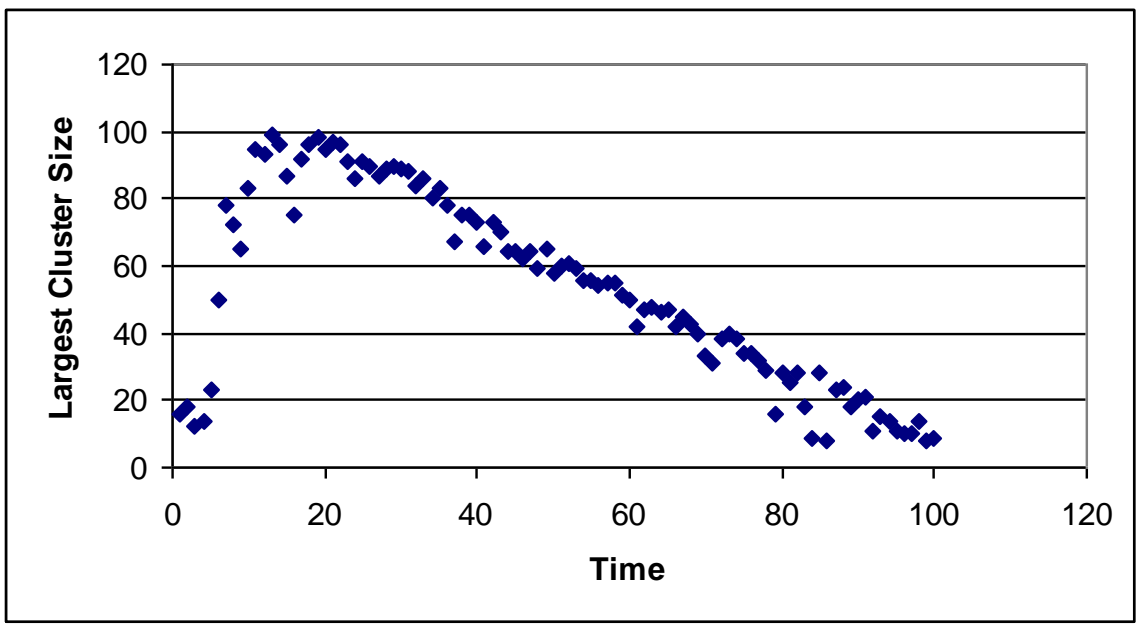

Figure 2: Evolution of the Largest Red Cluster Size within the Scenario (Red Unsuccessful).

When Red is unsuccessful, only the first two phases are visible, as shown in Figure 2. (Note all plots of clustering consider only the agents, which are still alive at the moment of sampling).

Let us look now at the spectrum of cluster size. The Red agents are able to generate a wide range of different cluster sizes. This can be seen by examining the frequency plot of the largest cluster size, over a replication of the scenario. Figure 3 shows two representative plots, for two replications of the scenario corresponding to cases where Red was successful (replication 1) and Red was unsuccessful (replication 40).

For the replications of the scenario where Blue was unsuccessful in its defence of the high value objective, Blue only generated a narrow spectrum of largest cluster size across the time evolution of the scenario. However, for the singular case where Blue was successful, Blue was able to generate a wider spread. This is illustrated in Figure 4, where the narrow spread of largest cluster size is shown for a number of unsuccessful Blue replications, and compared to the singular case (replication 40) where Blue was successful. 


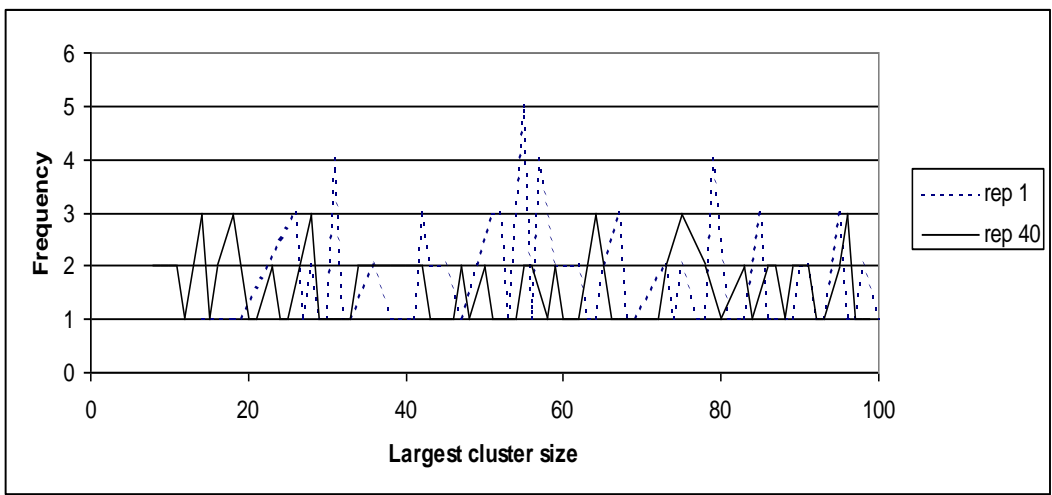

Figure 3: Frequency Distribution of Largest Red Cluster Size.

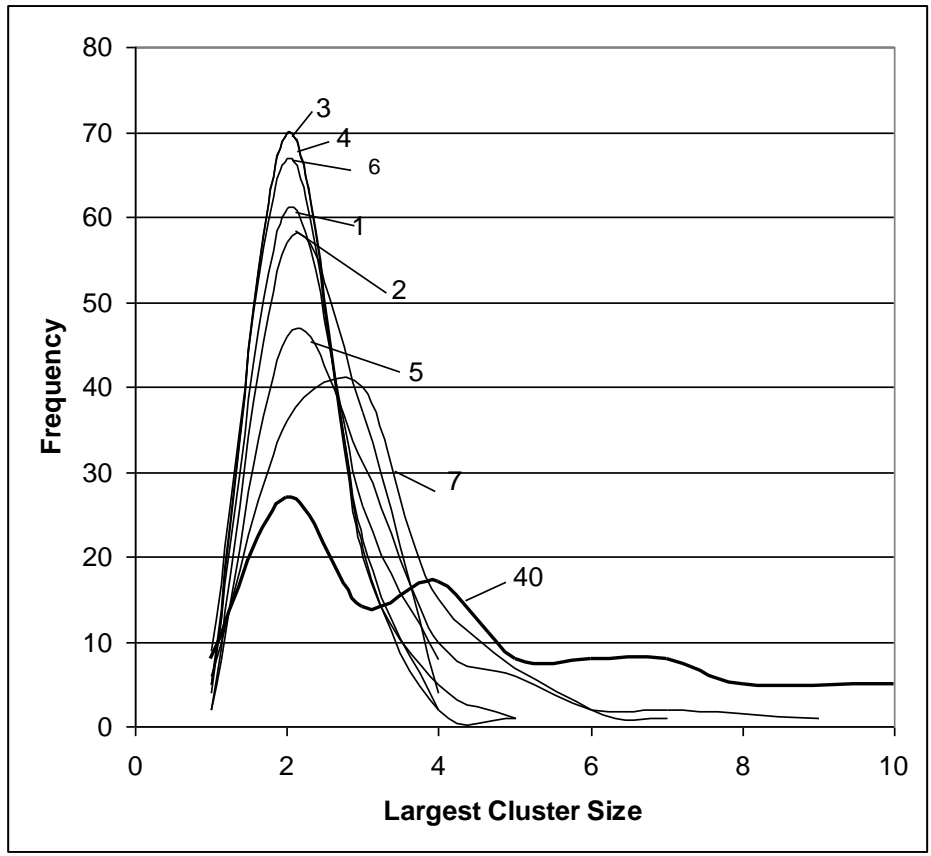

Figure 4: Frequency Distribution of Largest Blue Cluster Size. 


\section{Cluster Size Distribution}

All of this experimental evidence, based on looking just at the largest cluster size at a given time step in the simulation, indicates that clustering is a key component in determining the emergent behaviour of the agent simulation, as is also predicted by our previous theoretical analysis. ${ }^{21}$ Let us now look at the statistical distribution across all cluster sizes, averaged across a scenario replication. From theory we expect this to be a power law distribution, i.e. of the form:

$$
P(S): S^{\alpha}
$$

where $S$ is the cluster size, and $\alpha$ is a power law exponent. On a Log-Log scale, this implies a straight-line relationship. We would expect to see 'cut-off effects' at each end of such a plot where the relationship breaks down due to finite scaling effects (for example we cannot consider a cluster size smaller than 1). Figure 5 shows such a Log-Log plot of cluster size for the Red agents, over a number of replications. It is clear from this that there is such an intermediate regime of system behaviour, as expected from theory, where the cluster size distribution follows a power law distribution. This is indicative of a fractal relation for cluster creation. ${ }^{22}$

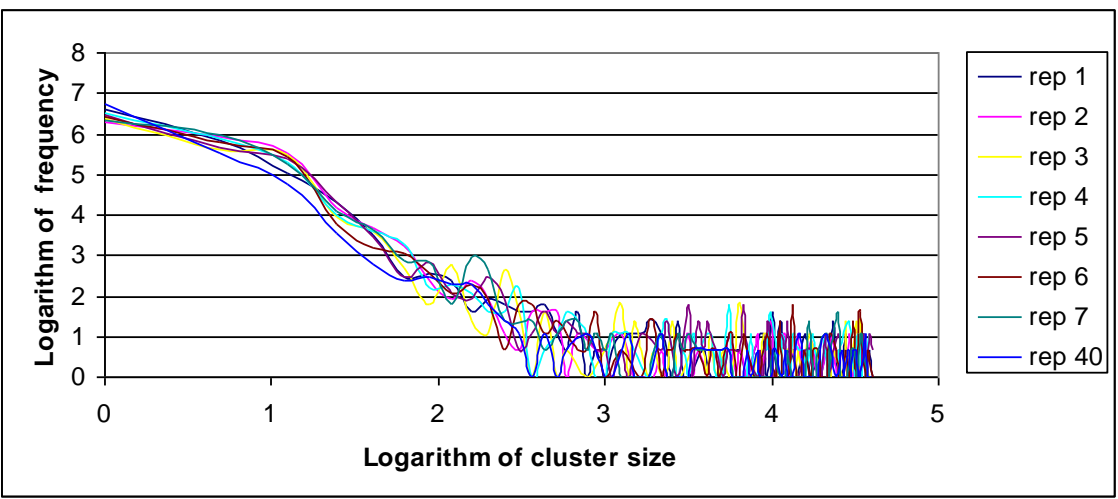

Figure 5: Distribution across All Red Cluster Sizes. 


\section{Conclusion}

We have first shown in detail how, for certain types of conflict across a frontage, the dynamics of the conflict evolves toward a fractal attractor whose fractal dimension lies in the range (from historical experimental data) of 1.5-1.685. This is shown to be compatible with results of the cellular automata model of 'invasion percolation' that produces a fractal front with dimension in the range 1.33-1.89. We have then shown that clustering is a key contributor to the emergent behaviour of the ISAAC cellular automata model of conflict. The nature of this contribution is as expected from our earlier theoretical analysis. In particular, the distribution of cluster size follows a power law in the intermediate regime; a signal of fractal clustering.

\section{Notes:}

James Moffat and Susan Witty, "Phase Changes in Meta-Modelling Using the Fractal Dimension," Information and Security: An International Journal 8, 1 (October 2002): 52-67.

2 David S. Alberts, John J. Garstka, and Frederick P. Stein, Network Centric Warfare; Developing and Leveraging Information Superiority, $2^{\text {nd }}$ Edition (Washington DC: CCRP, DoD, USA, 2000); David S. Alberts, John J. Garstka, Richard E. Hayes and David A. Signori, Understanding Information Age Warfare (Washington DC: CCRP, DoD, USA, 2001); James Moffat, Command and Control in the Information Age Representing its Impact (London, UK: The Stationery Office, 2002).

3 James Moffat, Complexity Theory and Network Centric Warfare (Washington DC: CCRP, DoD, USA, 2003), <http://www.dodccrp.org/publications/pdf/Moffat_ Complexity.pdf> (12 Feb. 2004).

4 James Moffat and M. Passman, "Metamodels and Emergent Behaviour in Models of Conflict," Simulation Modelling Practice and Theory (in press); Moffat, Complexity Theory and Network Centric Warfare.

5 Maya Paczuski, Sergei Maslov, and Per Bak, "Avalanche Dynamics in Evolution, Growth, and Depinning Models," Physics Review E 53, 1 (January 1996): 414-443; Henrik J. Jensen, Self-Organised Criticality (Cambridge, UK: Cambridge University Press, 1998).

6 Moffat, Complexity Theory and Network Centric Warfare.

7 Michael K. Lauren, "Modelling Combat using Fractals and the Statistics of Scaling Systems," Military Operations Research 5, 3 (2000): 47-58.

Andrew Ilachinski, "Irreducible Semi-Autonomous Adaptive Combat (ISAAC): An Artificial-Life Approach to Land Combat," Military Operations Research 5, 3 (2000): 29-46.

9 < http://www.mcwl.quantico.usmc.mil/divisions/albert/index.asp > (19 October 2003).

Arun V. Holden, ed., Chaos Non-Linear Science; Theory and Applications (Manchester, UK: Manchester University Press, 1986). 
Moffat, Complexity Theory and Network Centric Warfare.

David Rowland, "The Effectiveness of Infantry Small Arms Fire in Defence - A Comparison of Trials and Combat Data," unpublished DOAC Memorandum (1983).

J. H. Thody and H. J. Dove, "An Analysis of Small Arms Fire by Infantry in Defensive Positions," DOAE Unpublished Note (1981).

David Rowland, personal communication.

Rowland, personal communication.

David Rowland, "The Effect of Combat Degradation on the Urban Battle," Journal of the OR Society 42, 7 (1991): 543-553.

\section{David Rowland, personal communication.}

See the Appendix of Paczuski, Maslov, and Bak, "Avalanche Dynamics in Evolution, Growth, and Depinning Models."

J. Hoshen and R. Kopelman, "Percolation and Cluster Distribution. I. Cluster Multiple Labeling Technique and Critical Concentration Algorithm," Physics Review B 1, 14 (October 1976): 3438-3445.

Moffat and Witty, "Phase Changes in Meta-Modelling Using the Fractal Dimension;" Moffat, Complexity Theory and Network Centric Warfare.

Discussed in more detail in Moffat, Command and Control in the Information Age Representing its Impact; Paczuski, Maslov, and Bak, "Avalanche Dynamics in Evolution, Growth, and Depinning Models."

Professor JIM MOFFAT is a Fellow at the Defence Science and Technology Laboratory, UK, a Fellow of Operational Research and a visiting Professor at Cranfield University, UK. He was awarded the President's medal of the Operational Research Society for the year 2000. He holds a first class honours degree in Mathematics and a PhD in Mathematics, and was awarded the Napier medal in Mathematics by the University of Edinburgh. He has worked for the past 20 years on defence related operational analysis problems and aerospace technology research. His current research interest is in building analysis tools and models, which capture the key effects of human decision making and the other aspects of C4ISR. E-mail: jmoffat@dstl.gov.uk.

SUSAN WITTY has an honours degree in Mathematics and a PhD in aero-acoustics research. She has been an analyst in the Defence Science and Technology Laboratory for about four years. Her current research interests are complexity science and Bayesian networks applied to high-level data fusion. 